T. trichiura 1007 CAAGGAATCGGAAAGCTGCGACTGCCTGCAAGGGTTTCAGTTGACTCATTCCCTCGGCGGCGGAACTGGGAGTGGAAT Consensus_H.baboon Consensus_human

\title{
T. trichiura
} Consensus_H.baboon Consensus_human

.

167

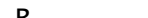

T. trichiura GAAGGCAAGTTGTTTGATACTGTTCACGTCGTGAACTATCGCCTTTTTAGGTTTCAGATACAGTTGTAGAACCATATA Consensus_H.baboon Consensus_human

198 200

T. trichiura Consensus_H.baboon Consensus_human

\section{ATGCAACTCTGTCAGTCCACCAGTTGGTAGAGAACACGGACGAAACATTCTGCATAGATAATGAAGCGCTTTACGATA}

T. trichiura TTTGTTTCCGAACTTTGAAGTTAACAACACCAACTTACGGAGACTTAAATCATTTGGTTTCGGCAACCATGTCTGGAG Consensus_H.baboon Consensus_human

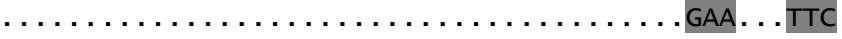

